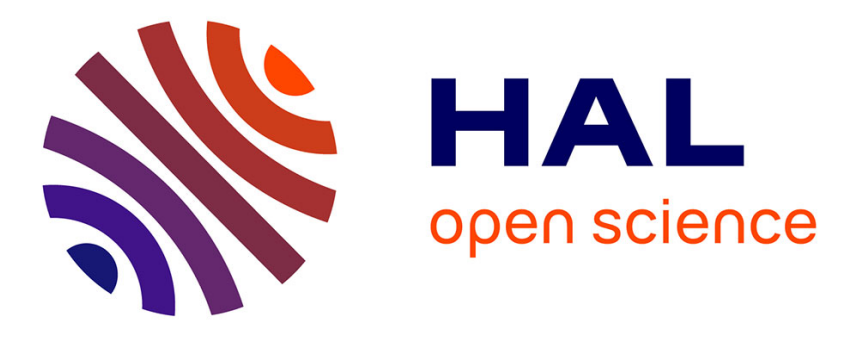

\title{
Introducing Linear Magnetic Materials in PEEC Simulations. Principles, Academic and Industrial Applications
}

Jean-Pierre Keradec, Edith Clavel, Jean-Paul Gonnet, V. Mazauric

\section{- To cite this version:}

Jean-Pierre Keradec, Edith Clavel, Jean-Paul Gonnet, V. Mazauric. Introducing Linear Magnetic Materials in PEEC Simulations. Principles, Academic and Industrial Applications. IAS 2005, Oct 2005, Hong Kong SAR China. hal-00185592

\section{HAL Id: hal-00185592 \\ https://hal.science/hal-00185592}

Submitted on 12 Feb 2009

HAL is a multi-disciplinary open access archive for the deposit and dissemination of scientific research documents, whether they are published or not. The documents may come from teaching and research institutions in France or abroad, or from public or private research centers.
L'archive ouverte pluridisciplinaire HAL, est destinée au dépôt et à la diffusion de documents scientifiques de niveau recherche, publiés ou non, émanant des établissements d'enseignement et de recherche français ou étrangers, des laboratoires publics ou privés. 


\section{Introducing Linear Magnetic Materials in PEEC Simulations. Principles, Academic and Industrial Applications}

\author{
J-P. Keradec (IEEE member), E. Clavel, \\ Laboratoire d'Electrotechnique de Grenoble \\ (LEG) -INPG/UJF-CNRS UMR 5529 \\ ENSIEG - Domaine Univertisitaire - BP46 \\ 38402 Saint-Martin-d'Heres cedex - France \\ Jean-Pierre.Keradec@,leg.ensieg.inpg.fr
}

\author{
J-P. Gonnet, V. Mazauric \\ Schneider Electric, Corporate R\&D \\ A2 Research Center, 9 quai Paul Louis Merlin \\ 38050 Grenoble Cedex 9 - France \\ jean-paul.gonnet@schneider-electric.com
}

\begin{abstract}
In order to study impact of wiring impedances PEEC simulation method has proved to be reliable, fast, and applicable to a wide range of practical problems. However, up till now, this method is inapplicable when a piece of magnetic material is located close to one of the studied wire.

In this paper we present a method that overcomes this limitation with a very little cost in computing time. Owing to it, magnetic materials can be introduced, assuming only their behavior is linear. They can be conductive or not and their permeability can be complex to account for magnetic losses.

Principles of the extension are carefully established and some academic problems are solved to compare with both analytical formulation and results of FEM simulation software. To end, an industrial problem is solved to illustrate the practical interest of this extension
\end{abstract}

Keywords- electromagnetic simulation, eddy currents, PEEC, wiring behavior.

\section{INTRODUCTION}

As soon as voltage droop along wires is not negligible regarding voltages normally handled by the circuit, controlling electrical behavior of wires becomes a key point to reach reliable working of the circuit. In power switching electronics this is of major concern because currents flowing in wires are high and, due to high switching speed, they includes high frequency content. As a result, wire impedances lead to high voltage drops that originate losses or, even, prevent proper working of converters.

Forecasting the whole behavior of an electronic circuit before building it is a topical challenge for most of engineers. To reach this goal, circuit simulation software is now so flexible and powerful that the main limitation of accuracy is the availability of adequate models for each component. During the last two decades, decisive progresses have been made in representing every kind of active and passive components. Among the last ones, special attention has been paid to wiring.
In power electronics wire length is often far shorter than involved wavelength, even for highest harmonics of working frequencies. As a result, wiring is efficiently split in two: inductive wiring and stray capacitances. These two parts intervene in parallel so, on the low frequency side, inductive wiring dominates. According to this, inductive wiring can be studied separately and, from low to medium frequency, it brings the major contribution to wiring influence. In practice, inductive wiring has two major impacts. First, it induces series voltages that can prevent proper working of the circuit, second, due to eddy currents, series power losses are sometime dramatically increased (fig. 1). These two phenomena caused a large number of industrial studies (fig. 2) [1-2].
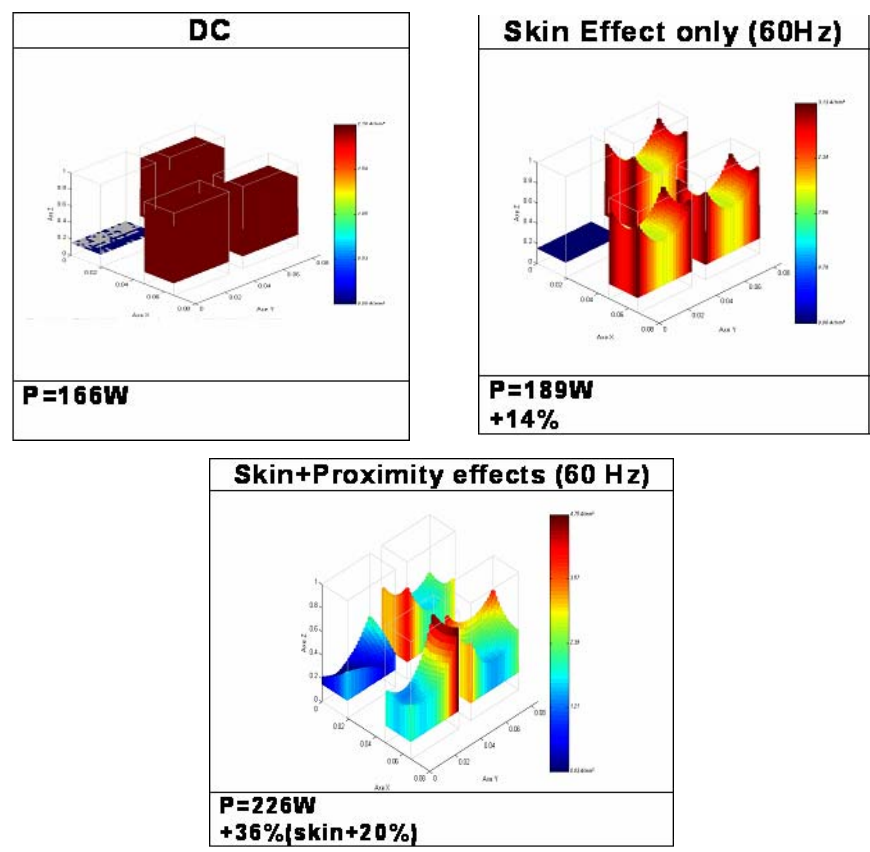

Figure 1. Impact of eddy currents on busbar series losses. 

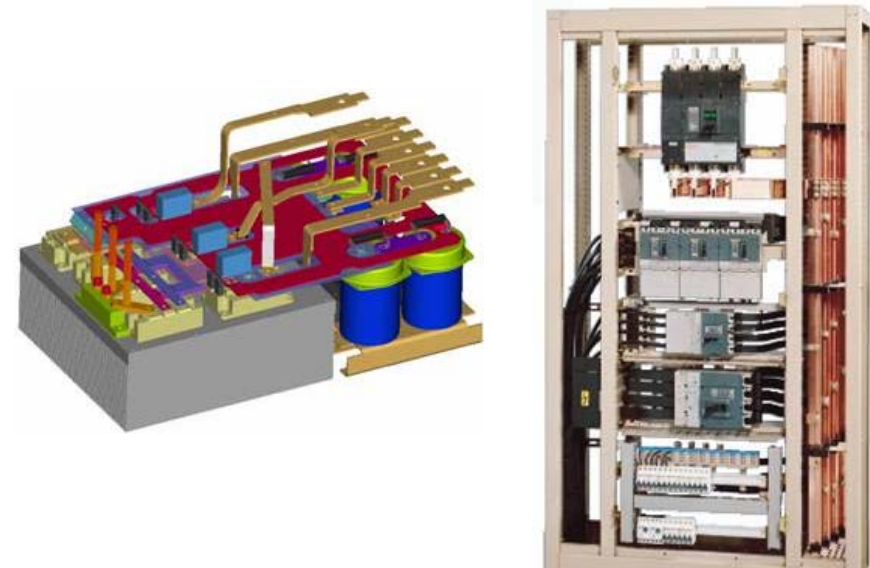

Figure 2. Devices for which impact of wiring cannot be neglected. a) Power electronics converter, b) Switchboard panel including a $50 \mathrm{~Hz} 3$-phased distribution busbar

To study the contribution of inductive wiring, every wire or piece of wire of the circuit is considered as a weak inductance that is coupled with every other one. Of course, real wires are also resistive. To summarize, one can says that inductive wiring is fully described by an inductance matrix which accounts for losses.

Three methods allow the matrix impedance to be evaluated: analytical calculation, FEM simulation and PEEC computation. First one is unusable when system becomes complicated (fig. 2 ), but the two others are efficient. They both allow various electrical and mechanical parameters to be evaluated. As soon as an optimization process is to be run to solve an industrial problem [3], computation time becomes a key point to choose. From this point of view, PEEC method, which doesn't need air to be meshed, is far faster. Unfortunately, up till now, this method was not applicable to devices including magnetic materials. Such a situation is frequent because converters include coils and transformers with magnetic core and, in some applications, influence of iron based enclosure or shielding must be taken into account.

The main purpose of this paper is to present a method that overcomes this limitation.

We first remind basic assumptions on which standard PEEC method relies. Then, we consider an extension to inhomogeneous magnetic materials. In this situation, a new formulation is established to compute vector potential. In the particular case where relative permeability equals 1 except in a volume $\mathrm{V}$ where it equals $\mu \mathrm{r}$, vector potential computation appears to be quite simple and result looks similar to Ampere's equivalent currents. These two formulations are compared and surface currents are justified. We finally deduce the matrix formulation which has been introduced in our software.

To conclude, the new formulation is checked on two simple academic problems that are connected to industrial ones.

\section{BASE OF STANDARD PEEC METHOD}

\section{A. Physical base of the method.}

Let us consider a linear, homogenous and isotropic (lhi) material. If fields are sinusoidal versus time, and if charge density and displacement current are negligible, Maxwell equations are written as follows.

$$
\begin{array}{ll}
\overrightarrow{\operatorname{rot}} \overrightarrow{\boldsymbol{H}}=\vec{J} & \text { (1) } \overrightarrow{\operatorname{rot} \vec{E}}=-\frac{\partial \overrightarrow{\boldsymbol{B}}}{\partial t} \\
\operatorname{div} \overrightarrow{\boldsymbol{B}}=0 & \text { (3) } \quad \operatorname{div} \vec{E}=0
\end{array}
$$

Material behavior is described by the two following equations.

$$
\overrightarrow{\boldsymbol{J}}=\sigma \overrightarrow{\boldsymbol{E}} \quad \text { (5) } \quad \overrightarrow{\boldsymbol{B}}=\mu_{0} \overrightarrow{\boldsymbol{H}}
$$

These 6 equations fully describe all the electromagnetic behavior of the considered medium. Potentials are then introduced according to the following equations:

$$
\begin{aligned}
& \vec{B}=\overrightarrow{\boldsymbol{r o t} t} \vec{A} \\
& \text { (7) } \quad \operatorname{div} \vec{A}=0 \\
& \vec{E}=-\overrightarrow{\operatorname{grad}} \boldsymbol{U}-\frac{\partial \overrightarrow{\boldsymbol{A}}}{\partial t}
\end{aligned}
$$

Joining (7), (6) and (1) leads to Poisson's vector equation:

$$
\Delta \overrightarrow{\boldsymbol{A}}=-\mu_{0} \overrightarrow{\boldsymbol{J}}
$$

Similarly, joining (9), (4) and (8) leads to Laplace's scalar equation:

$$
\Delta \boldsymbol{U}=0
$$

Moreover, owing to space Fourier transform, from (10) and (8) it comes [ ]:

$$
\vec{A}(\vec{r})=\frac{\mu_{0}}{4 \pi} \int_{\text {espace }} \frac{\overrightarrow{\boldsymbol{J}}\left(\vec{r}^{\prime}\right)}{\left|\vec{r}-\vec{r}^{\prime}\right|} d v
$$

With $\overrightarrow{\boldsymbol{A}}$ expressed as (12), relations (1), (3), (6), are automatically satisfied and, with $\boldsymbol{U}$ given by (9), (2) is also satisfied. Now, assuming all time variations are harmonic, we group (5) and (9):

$$
-\overrightarrow{\operatorname{grad}} U=\frac{1}{\sigma} \vec{J}+j \omega \vec{A}
$$

We can now forget all equations but (12), (13) and (4) because, if these three ones are satisfied, equations (1) to (6) are satisfied too.

\section{B. Matrix writting.}

PEEC is used when volumes in which currents flow can be divided in elementary thin straits wires. In such a wire, current density is assumed to be parallel to the length of the wire and to be constant in the whole volume of this wire. In such a 
situation, vector potential is computed by adding the contributions of all these elementary sources.

According to these assumptions, studied problem becomes a linear circuit problem, in which the number of ports equals that of elementary wires. Involved currents $\boldsymbol{I}$ and voltages $\boldsymbol{V}$ are respectively that flowing in and that sustained by each of these wires. These variables are linked by impedance matrix $\boldsymbol{Z}$. To compute $\boldsymbol{Z}$ elements, one can fix independently all components of $\boldsymbol{I}$ and, then, evaluates $\boldsymbol{V}$. Equation set (14) is equivalent to (12), (13) and (4).

$$
\begin{aligned}
& \|\boldsymbol{V}\|=\|\boldsymbol{Z}\| \cdot\|\boldsymbol{I}\| \\
& \boldsymbol{Z}=\left[\begin{array}{c}
\boldsymbol{R}_{i} \\
0
\end{array}\right]+j \omega\left[\begin{array}{c}
\boldsymbol{M}_{i j} \\
\boldsymbol{M}_{i j}
\end{array}\right] \\
& =\frac{\mu_{0}}{4 \pi} \int_{C i} \int_{C j} \frac{1}{\left|\vec{r}-\vec{r}^{\prime}\right|} \overrightarrow{d l}_{i} \overrightarrow{d l}_{j}
\end{aligned}
$$

As soon as impedance matrix $\boldsymbol{Z}$ is known, that describing the real wiring can be deduced because voltages input in the real circuit are sums of calculated voltages while associated currents are sums of calculated currents. These connections in series or in parallel give the impedance matrix describing the real circuit wiring which owns far less ports.

\section{EXTENSION TO $\mu$ PEEC METHOD}

\section{A. Extension to an inhomogeneous magnetic media}

In a linear isotropic magnetic medium, among local equations (1) to (6), only (6) must be modified: a scalar permeability $\mu_{r}$ must be introduced. If field time variations are sinusoidal, accounting for losses only needs considering $\mu_{r}$ is complex.

$$
\overrightarrow{\boldsymbol{B}}=\mu_{0} \mu_{r} \overrightarrow{\boldsymbol{H}}
$$

If the medium is homogeneous, ( $\mu_{r}$ identical in whole space), this coefficient multiplies the current density of (10) everywhere so it also multiplies (12).

$$
\overrightarrow{\boldsymbol{A}}_{1}(\vec{r})=\frac{\mu_{0}}{4 \pi} \int_{\text {espace }} \frac{\mu_{r} \overrightarrow{\boldsymbol{J}}\left(\vec{r}^{\prime}\right)}{\left|\vec{r}-\vec{r}^{\prime}\right|} d v
$$

Usually, one says linked currents $\overrightarrow{\boldsymbol{J}_{\text {link }}}$ reinforce free currents $\overrightarrow{\boldsymbol{J}_{\text {free }}}$. This is consistent if, everywhere:

$$
\overrightarrow{\boldsymbol{J}_{\text {link }}}=\left(\mu_{r}-1\right) \overrightarrow{\boldsymbol{J}_{\text {free }}}
$$

When the medium is inhomogeneous, rotational calculation introduces an extra term.

$$
\begin{aligned}
\overrightarrow{\boldsymbol{r o t}}\left(\mu_{0} \mu_{r} \overrightarrow{\boldsymbol{H}}\right) & =\mu_{0} \overrightarrow{\boldsymbol{r o t}}\left(\mu_{r} \overrightarrow{\boldsymbol{H}}\right)=\mu_{0}\left(\mu_{r} \overrightarrow{\operatorname{rot}} \overrightarrow{\boldsymbol{H}}-\overrightarrow{\boldsymbol{H}} \wedge \overrightarrow{\operatorname{grad}} \mu_{r}\right) \\
& =\mu_{0} \mu_{r} \overrightarrow{\boldsymbol{J}}-\mu_{0} \overrightarrow{\boldsymbol{H}} \wedge \overrightarrow{\operatorname{grad}} \mu
\end{aligned}
$$

In this case, Poisson's equation (10) becomes:

$$
\Delta \overrightarrow{\boldsymbol{A}}=-\mu_{0} \mu_{r} \overrightarrow{\boldsymbol{J}}+\mu_{0} \overrightarrow{\boldsymbol{H}} \wedge \overrightarrow{\operatorname{grad}} \mu_{r}
$$

So, $\overrightarrow{\boldsymbol{A}}$ results from two contributions. First one (16) is easy to explain: comparison of (10) and (15) shows that $\mu_{r} \vec{J}$ vector has replaced $\overrightarrow{\boldsymbol{J}}$. Vector potential due to this contribution is obtained by making the same replacement in (12) that leads to (16). Second contribution, which is written as (19), is more difficult to justify in the general case.

$$
\overrightarrow{\boldsymbol{A}_{2}}(\vec{r})=\frac{\mu_{0}}{4 \pi} \int_{\text {espace }} \frac{-\overrightarrow{\boldsymbol{H}} \wedge \overrightarrow{\boldsymbol{g r a d}} \mu_{r}}{\left|\vec{r}-\vec{r}^{\prime}\right|} d v
$$

To go further, we now restrict our study to cases in which space is divided in homogeneous magnetic regions, each characterized by its own permeability.

\section{B. Piece of magnetic material in air}

So let us consider a system in which only a volume $\mathrm{V}$, limited by surface $\mathrm{S}$, of homogenous magnetic material of permeability $\mu_{r}>1$ which is introduced in surrounding air. In this case, second term of (18) is null everywhere except on $\mathrm{S}$ and associated vector potential (19) can be written simply.

To reach this goal we consider permeability decreases from $\mu_{r}$ to 1 within a length $\varepsilon$ as short as wanted, with its gradient directed as the normal $\vec{n}$ to the surface $\mathrm{S}$. Integration domain is a thin layer which extends on both sides of $\mathrm{S}$ and the elementary volume obtained by multiplying the elementary surface $d s$ by $\varepsilon$.

$\boldsymbol{A}_{2}(\vec{r})=\frac{\mu_{0}}{4 \pi} \oint_{S} \frac{-\overrightarrow{\boldsymbol{H}} \wedge \overrightarrow{\boldsymbol{n}} \frac{1-\mu_{r}}{\varepsilon}}{\left|\vec{r}-\vec{r}^{\prime}\right|} \varepsilon d s=\frac{\mu_{0}}{4 \pi} \oint_{S} \frac{\left(\mu_{r}-1\right) \overrightarrow{\boldsymbol{H}} \wedge \overrightarrow{\boldsymbol{n}}}{\left|\vec{r}-\vec{r}^{\prime}\right|} d s$

Obviously, this contribution is due to a surface current $\overrightarrow{\boldsymbol{K}}$ and $\overrightarrow{\boldsymbol{A}_{2}}$ can be written as:

$$
\overrightarrow{\boldsymbol{A}_{2}}(\vec{r})=\frac{\mu_{0}}{4 \pi} \oint_{S} \frac{\overrightarrow{\boldsymbol{K}}}{\left|\vec{r}-\vec{r}^{\prime}\right|} d s
$$

$$
\text { With } \overrightarrow{\boldsymbol{K}}=\left(\mu_{r}-1\right) \overrightarrow{\boldsymbol{H}} \wedge \vec{n}
$$

\section{Why a surface current?}

Now, we will show that $\overrightarrow{\boldsymbol{K}}$ is exactly the current which allow linked current to stay enclosed in $\mathrm{V}$ without accumulating charges on $\mathrm{S}$.

Flux of current density through every closed surface is null. Let us consider a surface, half drop shaped, stuck on the internal face of S. Current density flux $I_{l}$ through this surface $\sigma_{1}$ is expressed in terms of $\overrightarrow{\boldsymbol{H}}$ : 


$$
I_{1}=\int_{\sigma_{1}} \overrightarrow{\boldsymbol{J}_{\text {lies }}} \overrightarrow{d s}=\left(\mu_{r}-1\right) \int_{\sigma_{1}} \overrightarrow{\operatorname{rot}} \overrightarrow{\boldsymbol{H}} \overrightarrow{d s}=\left(\mu_{r}-1\right) \oint_{c} \overrightarrow{\boldsymbol{H}} \overrightarrow{d l}
$$

If, besides, currents $\overrightarrow{\boldsymbol{J}_{S}}$ flow on $\mathrm{S}$ in a thickness $\varepsilon$, they go through surface $\sigma_{2}$ (Fig. 3) which have a base $\mathrm{C}$ and a height $\varepsilon$. They are equivalent to a surface current $\overrightarrow{\boldsymbol{K}}=\overrightarrow{\boldsymbol{J}_{S}} \varepsilon$. Moreover, $\overrightarrow{d l}$ being the elementary length on $\mathrm{C}$, the surface element of $\mathrm{S}$ is written as:

$$
\overrightarrow{d \sigma_{2}}=\vec{n} \wedge \overrightarrow{d l} \varepsilon
$$

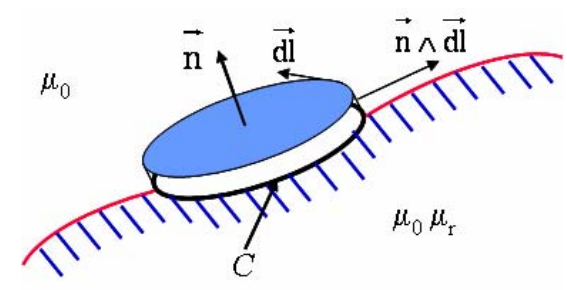

Figure 3: Surface currents on a magnetic material.

That leads to a new expression for $I_{2}$ :

$$
I_{2}=\oint_{C} \overrightarrow{\boldsymbol{J}_{s}}(\vec{n} \wedge \overrightarrow{d l} \varepsilon)=\oint_{C} \overrightarrow{\boldsymbol{K}}(\vec{n} \wedge \overrightarrow{d l})=\oint_{C}(\overrightarrow{\boldsymbol{K}} \wedge \vec{n}) \overrightarrow{d l}
$$

Having in mind that $I_{1}+I_{2} ;=0$, whatever be curve $\mathrm{C}, \overrightarrow{\boldsymbol{K}}$ is expressed as a function of $\overrightarrow{\boldsymbol{H}}$ :

$$
\begin{aligned}
& \vec{n} \wedge \overrightarrow{\boldsymbol{K}}=\left(\mu_{r}-1\right) \overrightarrow{\boldsymbol{H}} \\
& (\vec{n} \wedge \overrightarrow{\boldsymbol{K}}) \wedge \vec{n}=\overrightarrow{\boldsymbol{K}}(\vec{n} \vec{n})-\vec{n}(\vec{n} \overrightarrow{\boldsymbol{K}})=\overrightarrow{\boldsymbol{K}}=\left(\mu_{r}-1\right) \overrightarrow{\boldsymbol{H}} \wedge \vec{n}
\end{aligned}
$$

Where equation (22) has been multiplied, at right, by $\vec{n}$. This result is consistent with Ampere's current equivalence but it has been established following a different approach which accounts, from the beginning, for resistivity consequences and which clears up the role of surface currents.

\section{Matrix formulation}

The previous study shows that the major change to do when magnetic materials are involved consists in introducing surface currents. These currents avoid charge accumulation on S. In this view, we introduce surface elements (Fig 4).

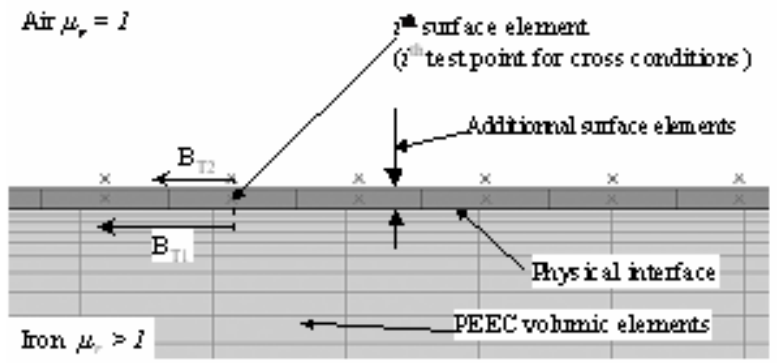

Figure 4: Surface elements are introduced, during meshing, on every magnetic material surface.
Now in a first step, for each elementary source of field $\boldsymbol{I}_{\mathrm{i}}$, we compute $\overrightarrow{\boldsymbol{A}_{1}}$ according to (16). In a second step, for this source of field, surface currents are adjusted so there is no discontinuity of the tangential part of $\overrightarrow{\boldsymbol{H}}$ in any location of S. Surface currents are indexed by $\mathrm{j}$ which varies according to their location and they all are proportional to elementary source of field $\boldsymbol{I}_{\mathrm{i}}$. For each value of $\mathrm{i}$ there is a set of value

$$
\boldsymbol{K}_{i}^{j}=\frac{1}{\boldsymbol{I}_{i}}\left(\frac{\boldsymbol{B}_{T}\left(x_{j}, y_{j}\right)}{\mu_{0} \mu_{r}}-\frac{\boldsymbol{B}_{T}\left(x_{j}, y_{j}\right)}{\mu_{0}}\right)
$$

Despite we consider only elementary source of field $\boldsymbol{I}_{\mathrm{i}}$, in (24) $\boldsymbol{B}_{T}$ is the field due to $\boldsymbol{I}_{\mathrm{i}}$ and all surface currents due to him. In a more compact formulation, this condition is written as:

$$
[\boldsymbol{K}] \cdot[\boldsymbol{I}]=0
$$

Then, all mutual induction between two elementary wires are deduced taking surface currents into account.

Finally, only slight changes are needed to change from standard PEEC to $\mu$ PEEC and increase in computation cost is low.

\section{APPLICATIONS}

Figure 5 shows calculation result for a rectangular piece of iron located between to opposite current conductors. Here, current densities in both conductors are uniform, so applied field is almost uniform. Parameters taken for iron are $\mu \mathrm{r}=1000$ and $\rho=10^{-8} \Omega \mathrm{m}$, frequency is $50 \mathrm{~Hz}$. Comparison with Flux $3 \mathrm{D}$ simulations are within a few percent regarding local induction values as well as power losses.
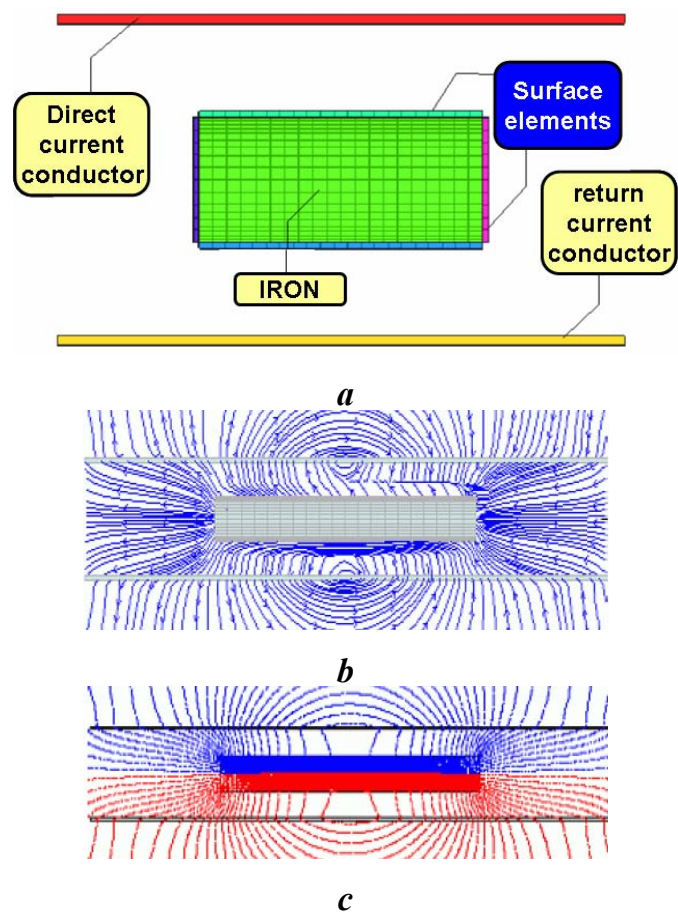

Figure 5. Iron barrel between two plane conductors. a) System description, b) mPEEC simulation, c) FEM simulation 
Second example addresses an asymmetric line: one wire is made of copper, other one is in magnetic material. With realistic permeability $(>1000)$, simulated induction shows a very large range of values so, verifications are not easy on a linear scale. It is the reason why we choose a low value $(\mu \mathrm{r}=10)$.
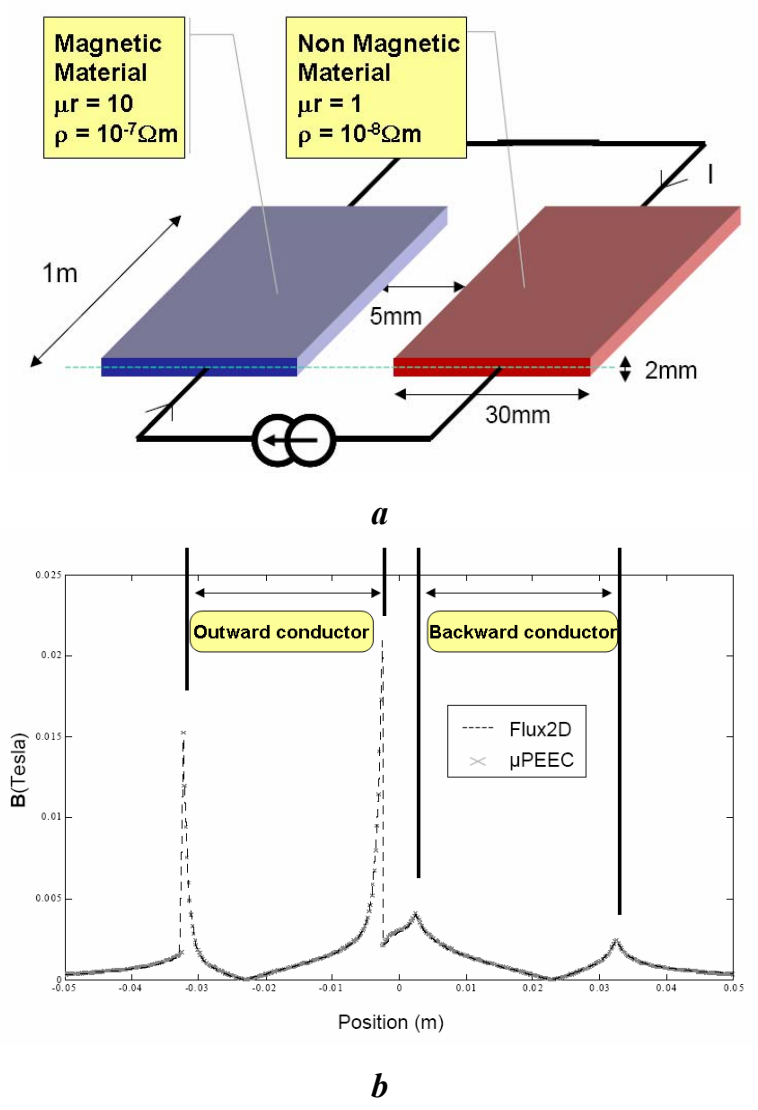

Figure 6: asymmetric line. a) system parameters b) comparison of Flux 2D and $\mu$ PEEC simulations
Curve shown in figure 6 is the modulus of the vertical component of induction. Not only $\mu$ PEEC results are in good agreement with Fem ones, but also discontinuity of tangential component of $\mathrm{B}$ is checked successfully: discontinuity ratio equals $\mu \mathrm{r}(10)$.

\section{CONCLUSION}

An extension of PEEC method, we called $\mu$ PEEC, is introduced in order to account for linear magnetic materials. This method requires linear and isotropic behavior of all involved magnetic materials but it applies if materials are conductive or not, even if several kinds are close together.

Bases of this approach are carefully established. Main results are consistent with Ampere's currents equivalence but chosen presentation allows wider extension. However, various devices have already been studied successfully.

Known applications of PEEC, including impedance and forces calculations, remain possible with $\mu$ PEEC and a lot of new ones are waiting to be checked. Among them, calculation of magnetizing and leakage inductances of ferrite core transformers.

\section{REFERENCES}

[1] [1]E. Clavel, J. L. Schanen, J. Roudet, A. Fontanet, "Influence of the Cabling Geometry on Paralleled Diodes in a High Power Rectifier", IEEE - IAS'96, San Diego CA, 6-10 Oct. 1996, pp 993 - 998

[2] [2]E. Clavel, J. Roudet, J-L. Schanen, P. Hublier, « Modeling and Electrical Simulation of a Busbar », IEEE - PCIM'96, Nuremberg, p 747-752, 21-23 may 1996.

[3] [3] J. P. Gonnet, J. M. Guichon, E. Clavel, Y. Maréchal, V. Mazauric, « Improving the energetic efficiency of distribution busbars », IEEE PES-TD, Dallas, USA, Sept. 2003

[4] [4]A. E. Ruehli, N. Rover, P. A. Brennan " Three dimensional inductance computations with partial element equivalent circuits », IBM Journal on R\&D, vol.23, ${ }^{\circ} 6$, Nov. 1979.

[5] Science, 1989. 